\title{
CAST OF CHARACTERS
}

Gesualdo Motta: Mastro-don Gesualdo

Mastro Nunzio Motta: his father

Santo Motta: his brother

Speranza: his sister, married to:

Massaro Fortunato Burgio

Uncle Mescalise: his uncle

Donna Bianca Trao: his wife

Don Diego Trao: Donna Bianca's brother

Don Ferdinando Trao: Donna Bianca's brother

Isabella Trao Motta: Gesualdo and Bianca's "daughter"

Aristocrats / Bianca's relatives

Baron Zacco

Baroness Zacco: his wife

Donna Lavinia: their daughter

Donna Marietta: their daughter

Baroness Rubiera: Donna Bianca's aunt

Don Nini (Antonino) Rubiera: her son

Donna Giuseppina Alosi: his wife

Don Filippo Margarone

Donna Bellonia: his wife

Donna Fifì

Donna Mita

their daughters

Donna Giovannina

Nicolino: their son

Donna Sarina (Sara) Cirmena: Donna Bianca's aunt

Corrado La Gurna: her ward

Donna Chiara Macrì: Donna Bianca's aunt

Donna Agrippina: her daughter 
xviii

Mrs. Marianna Sganci: Donna Bianca's aunt

Duke of Leyra (Alvaro Filippo Maria Ferdinando Gargantas di Leyra): Isabella's husband

Marina di Leyra: his sister and one of Isabella's schoolmates

Bali di Leyra: his uncle

Alimena: another of Isabella's schoolmates

Marquis Alfonso Limòli: Donna Bianca's uncle

Baron Mèndola

Others

Canon-priest Lupi

Archpriest Bugno

Father Angiolino: Donna Bianca's father confessor

Don Luca: sacristan

Grazia: his wife

Don Bastiano Stangafame: Captain at Arms and Donna Fifi's husband

Don Liccio Papa: head cop

Mr. Captain: Chief of Police

Mrs. Capitana: his wife

Fiscal Attorney

Notary Neri

Mommino Neri: his son

Notary Sghembi: from Militello

Doctor Tavuso: town doctor

Don Margheritino: his son and also a town doctor

Arcangelo Bomma: pharmacist

Doctor Muscio: one of Don Gesualdo's doctors

Don Vincenzo Capra: one of Don Gesualdo's doctors

Cavaliere Peperito 
Townspeople

Nanni l'Orbo: works for Don Gesualdo

Diodata: his wife; formerly Don Gesualdo's mistress

Nunzio: her son

Gesualdo: her son

Vito Orlando

Brasi Camauro

Agostino: overseer

Mastro Cola: mason

Massaro Carmine

Mariano: mason

Masi: helper boy

Mastro Nardo: mason

Mastro Lio Pirtuso: middleman and broker

Canali

Don Roberto Ciolla

Giacalone

Pelagatti

$\left.\begin{array}{l}\text { Alessio } \\ \text { Rosaria } \\ \text { Gerbido }\end{array}\right\} \quad$ work for Baroness Rubiera

Mastro Titta: Mrs. Sganci's house barber

Don Giuseppe Barabba: Mrs. Sganci's butler

Fra Girolamo of the Mercenaries: revolutionary cleric

Giacinto: sherbert shop owner

Nanni Ninnarò: inn owner

Mastro Cosimo: carpenter

Don Anselmo: a Caffè dei Nobili waiter

Signora Aglae: actress

Signor Pallante: actor

Don Leopoldo: Don Gesualdo's manservant in Palermo

Donna Carmelina: Isabella's wardrobe maid 
